\title{
Aproximación socio-antropológica a la obesidad infantil: estudio de caso en dos colegios de Valencia (España)
}

\author{
An anthopological-social approach to \\ childhood obesity: A case study in two \\ schools in Valencia (Spain)
}

Julia NAVAS LÓPEZ

José PALACIOS RAMÍREZ ${ }^{2}$

Asunción ROCA GEA ${ }^{3}$

RE S U M E N

\section{Objetívo}

El artículo analiza los marcos interpretativos de 82 niños/as (10-12 años) y de 10 madres/1 padre, sobre obesidad y sobrepeso, en dos colegios de Valencia (España).

\section{Métodos}

Se trata del estudio piloto de un proyecto de investigación cualitativa. Con los niños se usaron cuestionarios de respuesta abierta y elaboración de obras de teatro y cuentos, mientras que con los padres se realizaron grupos de discusión. Se tuvieron en cuenta aspectos como: diferencias de género, atributos negativos y formas de discriminación. En el caso de los padres, se propuso la cuestión de las causas y posibles soluciones del problema en relación a las existentes.

\section{Resultados}

Entre los niños/as emergieron términos negativos asociados al sobrepeso, presentando una mayor focalización corporal en el abdomen los niños y una notable importancia de la estética las niñas, unida a una percepción más amplia de los efectos del sobrepeso en la sociabilidad. Entre los padres, la preocupación tuvo una preponderancia en los efectos psicológicos del sobrepeso en sus hijos por encima de las consecuencias para la salud (solo en casos graves de obesidad).

\section{Conclusión}

La percepción de los padres sobre las posibles soluciones mostró la clara influencia de los discursos nutricionales, resultado de campañas de concienciación, así como el escaso conocimiento de otras posibles formas de intervención con mayor énfasis social o participativo.

Palabras-clave: Niño. Obesidad pediátrica. Padres. Percepción.

1 Universidad Católica San Antonio de Murcia, Departamento de Tecnología de los Alimentos y Nutrición. Campus Los Jerónimos,

s/n., 30107, Murcia, España. Correspondência con/Correspondence to: J NAVAS LOPEZ. E-mail: <jnavas@ucam.edu>

2 Universidad Católica San Antonio de Murcia, Departamento de Antropología Social y Cultural. Murcia, España.

${ }^{3}$ Dietista y Nutricionista. Murcia, España. 


\section{A B S T R A C T}

\section{Objective}

The article analyses the interpretive frameworks of 82 children (10-12 years) and 10 mothers/1 father about obesity and overweight in two schools in Valencia (Spain).

\section{Methods}

This is a pilot study of a qualitative research project. Children answered a questionnaire with open questions and asked to role play and create short stories. The parents participated in focus groups. The following aspects were taken into account: gender, negative attributes, and forms of discrimination. The parents were asked about the causes and possible solutions to the problem in relation to the existing ones.

\section{Results}

The children came up with negative terms for overweight. Boys were more focused on the abdomen and girls were more focused on aesthetics. Girls had a greater perception of the effects of overweight on sociability. The parents were more concerned with the psychological effects of overweight on their children than with its health effects (only in severe obesity cases). Translator note: I did not understand this sentence. Should it not be "except in severe obesity cases"?

\section{Conclusion}

Parents 'perception of possible solutions demonstrated a clear influence of the nutritional discourses stemming from awareness campaigns, as well as little knowledge of other possible interventions with greater social or participative emphasis.

Keywords: Child. Pediatric obesity. Parents. Perception.

\section{INTRODUCCIÓN}

La "Estrategia mundial sobre régimen alimentario, actividad física y salud" elaborada por la Organización Mundial de la Salud (OMS) ${ }^{1}$ manifiesta el interés de estudios sobre alimentación, salud y estilo de vida. Mientras que desde la ciencia se diversifican los puntos de vista, el grado de intervención en estos ámbitos es cada vez mayor. Tanto las estrategias políticas como los textos científicos apuntan en la misma dirección: la obesidad ha devenido una enfermedad de carácter epidémico y global, y hay que frenarla y evitarla.

En este trabajo se presentan los primeros resultados en el marco del proyecto titulado: "Una aproximación socio-antropológica a la obesidad infantil en tres estudios de caso: España, México y Cuba" (Plan Propio de Apoyo a la Investigación [PMAFI] 19/12).

Pese a estar cambiando, las investigaciones y programas de intervención sobre obesidad infantil suelen apoyarse en concepciones epidemiológicas vinculadas con metodologías antropométricas, frecuencias de consumo de alimentos y encuestas nutricionales ${ }^{2-4}$. Sin embargo, hace ya tiempo que las percepciones sociales de la obesidad y el sobrepeso son un tema de interés para la investigación, en el intento de mejorar las estrategias de prevención e intervención.

En ocasiones estas investigaciones presentan la percepción de los padres de niños con sobrepeso $u$ obesidad ${ }^{5}$, quienes parecen no percibir su impacto en la salud a medio-largo plazo o la percepción pública sobre la cuestión $n^{6}$. En otras, el interés se centra en las percepciones de la población adolescente sobre el problema y sobre las campañas de prevención $n^{7,8}$, por tratarse de una población especialmente sensible a los trastornos alimentarios y a los malos hábitos. Sin embargo, no muchos son los trabajos que se centran en la percepción de los niños que sufren el problema, y menos aun uniéndola con las de niños en cuyo caso no existe sobrepeso'. Lo que sí parece aceptado es que este tipo de estudios, más aun de corte cualitativo, están llamados a intentar mejorar la eficiencia de las campañas de prevención y concienciación. 
El objetivo del trabajo fue analizar los marcos interpretativos de niños/as de 10-12 años $\left(5^{\circ}-6^{\circ}\right.$ primaria) y de padres/madres en el contexto escolar y familiar, sobre obesidad y estilo de vida, teniendo en cuenta tanto la perspectiva de aquellos que presentaban problemas de sobrepeso como la de aquellos que no. Concretamente, se planteó analizar la definición y explicación del exceso de peso; recoger las diferencias interpretativas según el sexo; conocer la aparición de atributos negativos en relación a la obesidad y en qué medida esto provocaba implicaciones en procesos de discriminación, triangulando las perspectivas de infantes y padres. Además, en el caso de los padres, se pretende explorar las interpretaciones en torno a las causas de la obesidad y las posibles soluciones que se planteaban.

Se trata de un estudio piloto realizado en Valencia (España), que se incluye en la parte española de una investigación más amplia en España, México y Cuba. De manera colateral, la recogida de información nos permitió valorar la idoneidad de las técnicas elegidas.

\section{MÉTODOS}

La investigación se realiza en dos colegios, en el período de enero a julio de 2013 en Valencia (España). El trabajo de campo de tipo etnográfico ${ }^{10,11}$ incluyó una primera etapa de selección de los colegios. El interés previo mostrado por éstos y la consecuente facilidad para entrar en el aula-estudio fueron los criterios de selección. Se trabajó con niños/as - previo consentimiento de sus padres -, en $5^{\circ}$ y $6^{\circ}$ de primaria (10-12 años) y sus familias, en los colegios concertados de Les Carolines, en Picassent (Valencia) y María Inmaculada (Valencia). Fueron invitados a participar todos los niños/as, sin previo análisis antropométrico. La muestra la componían en Les Carolines 51 alumnos ( 25 de $5^{\circ}, 26$ de $6^{\circ}$ ) y 31 alumnos $\left(10\right.$ de $5^{\circ}, 21$ de $6^{a}$ ) en María Inmaculada. En el colegio María Inmaculada, algunos alumnos no recibieron la autorización de los padres. En una primera observación, estos alumnos podrían corresponder a alumnos con sobrepeso (no se realiza verificación antropométrica, pero la nutricionista obtiene información de la historia clínica de algunos excluidos). De esta forma, se prefirió la hora tras el recreo, solicitando al profesor que éstos pudiesen ser derivados a otras labores fuera del aula con la mayor discreción posible, para evitar que los demás alumnos pudieran relacionar estas ausencias con sus características físicas. Se optó por estas edades porque se consideró con capacidad para explicar conceptos y opiniones.

Se utilizó la observación participante en el aula, cuestionarios y narrativas (cuento y teatro). Los cuestionarios constaban de 14 preguntas abiertas, de las cuales 12 eran referidas al estilo de vida y 2 a la percepción corporal (en la pregunta 11 se pidió que se identificaran con una figura, dentro de una escala de 9 y con su correspondiente explicación) ${ }^{12}$.

Durante la observación participante se realizaron dos concursos en las categorías de narrativa: cuento y obra de teatro. No se les dio ninguna directriz más allá de que supieran que el contenido giraba en torno a la obesidad.

En el colegio Les Carolines, los alumnos escenificaron "Vamos a hacer un menú" en $5^{\circ} \mathrm{y}$ "Más vale tarde que nunca" en $6^{\circ}$. Ambos también elaboraron el guión. En el colegio María Inmaculada, la mayoría de los alumnos participantes redactaron, a modo individual, un cuento. En $5^{\circ}$ 4 cuentos y en $6^{\circ} 22$ cuentos.

A continuación se realizaron dos grupos de discusión con padres/madres voluntarios de ambos cursos. Fueron invitados todos los padres/ madres a través de una reunión previa con ellos en el Asociación de Madres y Padres (AMPA). Colegio Les Carolines: 3 madres y 1 padre del $5^{\circ}$ curso y 1 madre del $6^{\circ}$ curso; Colegio Maria Inmaculada: 6 madres del $6^{\circ}$ curso. Se lanzaron preguntas abiertas en torno al concepto de obesidad, estilos de vida, percepciones de los hijos y de posibles soluciones al problema, sin ofrecer información determinada a priori. Los datos recogidos se analizaron mediante el programa MAXQDA. 


\section{RESULTADOS Y DISCUSIÓN}

\section{Definición del exceso de peso}

En las respuestas de los niños/as al cuestionario predominó el uso del término gordo/ a, en ocasiones matizado por el diminutivo gordito/a, sobre todo en niñas, o "un poco de barriga", "michelines" en niños en alusión al abdomen. En el caso de los padres, en general matizaron el término gordo/a utilizando expresiones como "rechonchete" o "regordete" e siempre separando el sobrepeso, al que restaban importancia, vinculándolo con los costes psicológicos y sociales de la obesidad, que era vista como un problema grave de salud:

Habría que distinguir lo que es un exceso de peso, por alimentación, por genética, y una obesidad excesiva, porque una cosa es que te sobren 5 kilos, o tengas tendencia a engordar, pero cuando ya estás obeso, que se pasa, de que es enfermo, ya no sé si se puede solucionar con una buena alimentación o ejercicio, ya no sé si se puede llevar el cuerpo a su sitio (Grupo de discusión 1; informante: madre $\left.5^{\circ}\right)$.

Esto rompe parcialmente con la idea que ofrece la literatura, de que a menudo sobrepeso y obesidad son vistos como puntos de un continuum ${ }^{13,14}$, además de mostrar el calado de los discursos médicos y nutricionales sobre el problema en las convenciones de los padres.

\section{Diferencias en el marco interpretativo según sexo}

La literatura sobre el tema propone la idea de que, además de una condición corporal, el sobrepeso implica una experiencia psicológica y social, lo cual implicaría diferencias de sexo ${ }^{15}$. Así, aparecieron concepciones corporales distintas respecto al sobrepeso, en las que las niñas lo relacionaban con "barriga y piernas", mientras que los niños lo hacían solo con el abdomen. Es posible que estas diferencias incipientes puedan hallarse en la base de las diferencias en las prevalencias de las alteraciones del comportamiento alimentario ${ }^{16,17}$ y con el peso de la apariencia corporal en la vida social ${ }^{18}$.

\section{Atributos negativos y discriminación}

Ante las cuestiones: ¿Alguna vez te han llamado gordola? ¿Cómo te has sentido? ( $\left.n^{\circ} 12\right)$. Un 36\% respondió afirmativamente con similares proporciones de sexo. Es reseñable la mayor capacidad mostrada por las niñas para empatizar con el sentido negativo de la discriminación cuando no presentaban sobrepeso, o para referirse a ello figuradamente cuando sí era el caso. Solo una pequeña porción (12\%) respondió que dichos calificativos no les afectaban. En algunos casos, niños/as que no parecían presentar problemas de sobrepeso refirieron haber recibido calificativos negativos al respecto y haberse sentido mal.

Quizás fue en los cuentos y en las obras de teatro donde más claramente se observaron las connotaciones negativas asociadas al sobrepeso (Tablas 1 y 2). En general, se hacía referencia a una apariencia física no deseable vinculada a características sociales como la soledad, falta de amigos, incapacidad para los juegos de carácter físico y actitudes egoístas o descontroladas sobre la comida. Puede decirse que predominó el entendimiento del sobrepeso como un problema individual; algo que no es de extrañar, pues es el punto de vista (médiconutricional-psicológico) que predomina en las discursos científicos y sociales. Si las concepciones de los padres están impregnadas de ideas del conocimiento experto, igual sucede con los niños/ as, también receptores de cursos, actividades y otras formas de prevención que reflejan esta concepción individualizada:

En el caso de mi hija, si ve una niña que está gordita, dice: " pobrecita igual le pasa algo". Si es un $4 \times 4$, entonces si dice: "no 
Tabla 1. Fragmentos narrativas elaboración de cuentos: expresiones de desprecio. Valencia (Enero-Junio, 2013).

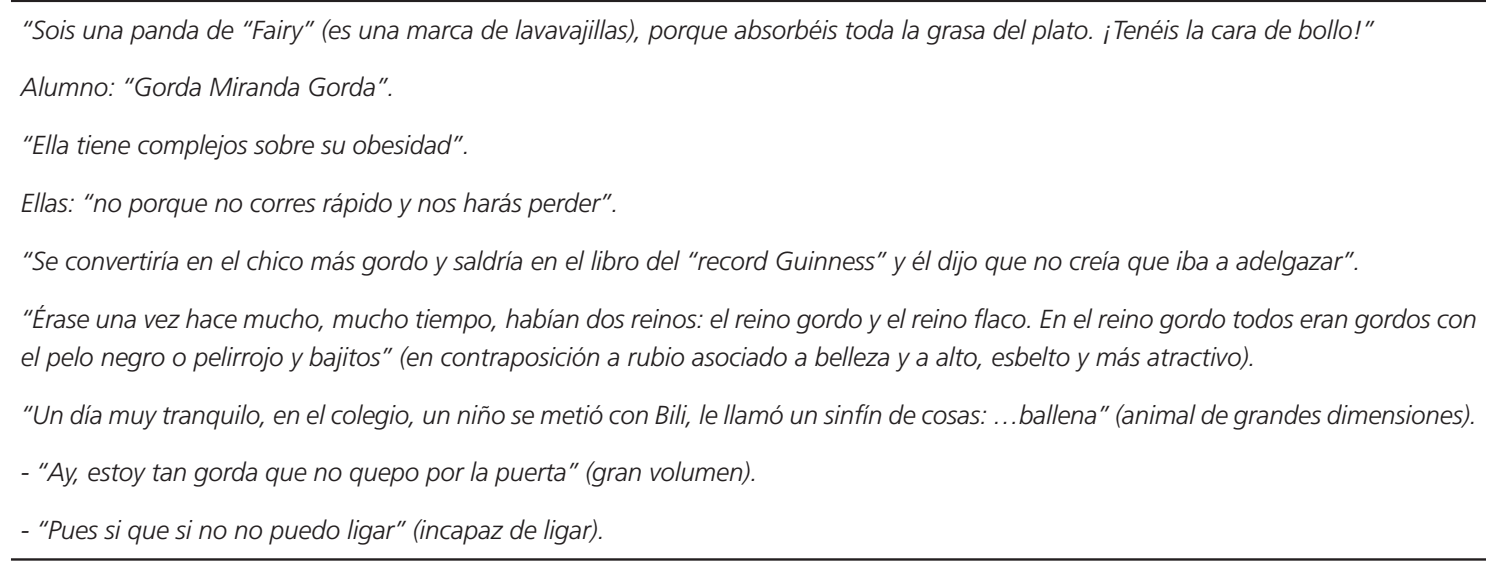

Tabla 2. Fragmentos narrativas sobre discriminación en 2 obras de teatro. Valencia (Enero-Junio, 2013).

"Marcos: No, vamos a jugar a ver quién se come tu bocadillo más rápido" (es un niño gordito) (A continuación comienzan a empujar a los dos compañeros delgados, pero Carlos y Lydia, al ser más rápidos, hacen unas piruetas y pueden huir del corro).

"Guille: ¡Eh, fideíto! Si no quieres algo me lo das. O mejor, me das todo ahora. Si no, me tiraré un pedo en tu cara" (es un niño gordito).

"Esther: el sobrepeso puede ser un problema de salud, debido a una falta de ejercicio, a una mala dieta o a un problema genético, y muchas veces se traduce en un problema estético, porque no encuentras tu talla, porque los demás no te ven bien".

"Miranda y Nicky: Nicky - porque te sientes sola, ¿acaso no tienes amigos? Miranda hizo un gesto con la cabeza diciendo que no".

"El chico, Carlos, pesaba 80 kg y todos sus amigos se reían de él, nadie quería estar con él" (narrador).

"Miguel era un niño de pocos amigos, por su obesidad. Después de tres años, este niño ha perdido muchos kilos y tiene muchos amigos, pero sobre todo ha ganado salud" (narrador).

"No podian estar juntos en flaquileño, como se les llamaba a los de flaco. Y un gordiense, como se llamaban a los de gordo" (narrador).

para de comer". Si ve una persona gorda, comiéndose un bocadillo de Nocilla, entonces dice:"se podría comer otra cosa a ver si así adelgazaba o se encontraba mejor... (Grupo de Discusión 2; Informante: madre $\left.6^{\circ}\right)$.

Uno de los aspectos que sorprendió, tanto en los cuestionarios como en los cuentos y obras de teatro, fue el valor de los juegos y actividades físicas entre las niñas, pues rompía ciertos estereotipos de sexo, reflejando parcialmente una tendencia homogeneizadora en la visión y efectos del sobrepeso en términos de sexo. Mientras tanto, los niños comienzan a preocuparse más por su apariencia (Tablas 3 y 4):
Ahora ya a los hombres o chicos, antes no tanto pero ahora creo que ya empieza a afectarles. Se van ellos cada vez se sienten peor, se van metiendo en su mundo, no se sienten tan admitidos a un grupo o a la sociedad, o al cole, o al grupo eso que son, y van ahí cambiando el carácter, se van haciendo más retraídos (Grupo de discusión 1; informante: madre $5^{\circ}$ ).

Las niñas comienzan a valorar ser activas y dinámicas, lo cual se refleja negativamente en el trato social que recibe alguien con sobrepeso: "Ellas: no porque no corres rápido y nos harás perder" (Narrativa: cuento; informante: niña de $6^{\circ}$ ). 
Tabla 3. Fragmentos cuestionario: Respuestas por sexo. Pregunta ¿Alguna vez te han llamado gordo/a?, ¿Cómo te has sentido? Valencia (Enero-Marzo, 2013).

\begin{tabular}{|c|c|}
\hline Niños & Niñas \\
\hline Si, muy mal. & Si, mal porque me han dicho que estoy obesa. \\
\hline Sí. Con rabia. & $\begin{array}{l}\text { Sí, porque mi hermana me llama gorda y me molesta porque no es } \\
\text { verdade. }\end{array}$ \\
\hline Si. Mal, porque como mucho. & Sí, pues mal. \\
\hline Si, mal. & Sí, pero lo decía de broma, así que no me molesto. \\
\hline $\begin{array}{l}\text { Sí, me daba igual porque los que lo dicen están más rechonchetes } \\
\text { que yo. }\end{array}$ & $\begin{array}{l}\text { No me han llamado porque yo soy delgada. Pero si me hubieran } \\
\text { llamado eso me sentiría muy mal. }\end{array}$ \\
\hline Si. Me he sentido mal. & $\begin{array}{l}\text { Una vez un compañero de clase me llamó ballena, me sentí un } \\
\text { poco mal, pero como yo sé que no lo estoy no me sentí tan mal } \\
\text { luego. }\end{array}$ \\
\hline Sí, pero yo paso de ellos aunque me sienta bastante mal. & Sí, mal, me molesta. \\
\hline Me llaman gordo de broma. & $\begin{array}{l}\text { No, nunca me han llamado eso, pero si me han llamado obesa, } \\
\text { pero de broma, aunque me molestó un poco, porque no estoy obe- } \\
\text { sa. }\end{array}$ \\
\hline $\begin{array}{l}\text { Sí, como siempre porque es una de la Educación Secundaria } \\
\text { Obligatoria. }\end{array}$ & $\begin{array}{l}\text { Si. Una o dos veces, pero por jorobar, jajaja. Me sentí un poco mal, } \\
\text { pero hago lo posible por rebajar. }\end{array}$ \\
\hline Si, me he sentido mal. & Si, no le doy mucha importancia pero a veces duele. \\
\hline Sí. Muy mal. & Sí. Pues mal porque a una persona le sienta muy mal. \\
\hline Si. Me he sentido normal ya que es mentira y lo sé. & No. Mal. \\
\hline Rubén. Mal. & No, me sentiría muy mal. \\
\hline Sí. Me ha dado igual. & Sí. No les hago caso. \\
\hline Sí. Mal. & $\begin{array}{l}\text { Si, pero gorda, no obesa. Tan mal que me puse a llorar. } \\
\text { Si. Pues mal porque fastidia y rebaja la autoestima. }\end{array}$ \\
\hline
\end{tabular}

En las discusiones de los padres emergen varias cuestiones importantes como la clara separación entre el sobrepeso, ligado a posibles secuelas psicológicas relacionadas con la estigmatización social de sus hijos y que muestra un claro trasfondo estético y moral de la cultura occidental moderna ${ }^{19-21}$, y la obesidad, que generalmente no suelen vincular con sus hijos, y que aparece como un problema de salud grave. Esto puede relacionarse con que las alteraciones de salud, fruto del sobrepeso, suelen aparecer posteriormente ${ }^{22}$.

Por otra parte los padres, especialmente con hijos con sobrepeso, mostraron una profunda preocupación por el hecho de que la investigación pudiera aumentar la estigmatización social de sus hijos (Tabla 5), lo cual muestra los efectos de responsabilización y culpabilización de las formas de definir el problema del sobrepeso y la obesidad desde instancias expertas ${ }^{23,24}$. Aunque hay que aclarar que las observaciones participantes en las aulas durante el desarrollo de los cuestionarios, las actividades de teatro y el cuento, no arrojaron información en este sentido:

Yo creo que él ahora está sufriendo, por todo esto, el teatro (Grupo discusión 1; informante: padre $6^{\circ}$ ).

Mi hijo cree que está gordo, de hecho esta mañana se ha puesto de perfil y ha dicho esto no lo quiero (el pecho), y es que tiene el esternón como su padre. Arnau, eres simpático, eres guapo, tus amigos te quieren un montón y esto no lo puedes quitar, porque tienes un cuerpo muy 
Tabla 4. Fragmentos grupo de discusión: Interpretaciones padres/madres diferencias percepción por sexo. Valencia (Marzo, 2013).

\begin{abstract}
"También pienso lo que dice Rosario, pero que no afecta igual al sexo masculino que al femenino, los niños no se lo toman igual que las niñas. A lo mejor según cierta edad, digo igual en alguna cosa sí, pero...".

"Ahora ya a los hombres o chicos, antes no tanto pero ahora creo que ya empieza a afectarles. Se van ellos cada vez se sienten peor, se van metiendo en su mundo, no se sienten tan admitidos a un grupo o a la sociedad, o al cole, o al grupo eso que son, y van ahí cambiando el carácter, se van haciendo más retraídos".

"En el ambiente de los críos, que a una persona la señalen de gordo o gorda, y hay diferencia entre chico y chica, le puede hundir. Lo que pasa es que va un poco con lo que decía antes, que depende cómo esa persona lleve la gordura o qué grado de gordura. Si es una obesidad que puede ser un indicio de que en un futuro vaya a generar problemas de salud, vale, pero si es del desarrollo, o de la constitución un poco más fuerte, o le cuesta más correr, porque es más patosa.... Suele utilizarse para machacar. Depende de la persona, porque a veces, una persona más gordita, no voy a decir obesa, porque tiene connotaciones más enfermizas, pues resulta que son más risueñas, más alegres. No sé si es una manera de subsanar para tener más amigos, no sé, suelen ser más graciosas".

"A las niñas les afecta más. La anorexia afecta más a las chicas. Son más miradas, más punto débil para meterse con ellas, están más condicionadas por la moda. La anorexia es una consecuencia de la gordura. A una chica gorda le han llegado a afectar tanto su autoestima que ha acabado en la anorexia".

"... Hay un reparto irracional de la comida. A las 11 se están comiendo un bocata enorme, es difícil que en la comida tengan hambre. Hay mucha diferencia entre chicos y chicas. En la comida los chicos comen rápido para ir al patio, las chicas más tranquilas en la mesa charlando de sus cosas. Las chicas están más condicionadas socialmente por su aspecto físico....los chicos también....las chicas más".

"Ellas... están más preocupadas por su aspecto: si están más gordas o menos gordas, si tienen más - tetas o menos... - en la ropa, que si mira lo que te has puesto... entre ellos también se miran y se miran el michelín... pero ellos luego se van a jugar, las chicas se quedan más rato con el tema".

"...los chicos también, los comentarios, ayyy, estás más blandito...".

"... mi hija se viste sin mirarse al espejo, y si se lo doy yo mejor, porque así no tiene que pensar, y el que tiene 7 años se peina en el espejo de la entrada, se mira como lleva el pelo. Vivimos en una sociedad que la imagen es tan importante, te bombardean por todos los sitios...".
\end{abstract}

Tabla 5. Repercusiones de la investigación en los niños/as en opinión de padres/madres. Valencia (Marzo, 2013)

"El otro día me dijo, creo que estoy un poco gordo. Hay que hablar con ellos".

"Yo le digo que está guapísimo y estupendísimo, que es como su papá. Y su papá pelea mucho con la comida, y no adelgaza, por eso yo estoy poniendo mucho hincapié en el tema genético, porque yo no digo que no le permitas un capricho de una golosina, también digo que come más que su hermano, supongo que ahora cuando retire un poco de comida creo que adelgazará. Creo que cuando se estiren... me faltaba ahora a mí esto".

parecido a tu padre. Yo creo que ahora se está dando cuenta de que tiene amigos más delgaditos y más gorditos, y más ahora con el tema este (los cuestionarios y el teatro), el está más ahí... (Grupo discusión 2; informante: madre $6^{\circ}$ ).

\section{Creencias sobre causas y soluciones propuestas}

En los modelos explicativos del sobrepeso y la obesidad narrados por los padres predominó una concepción nutricional del problema, marcada por el supuesto desequilibrio entre ingesta y gasto calórico (alimentación 121 referencias, actividad física 40) y quedando muy por detrás otras posibles causas más profundas como aspectos sociales (9 referencias), genéticos (8 referencias) o psicológicos (7 referencias). Algo que concuerda con la idea de que abordajes nutricionales y tratamientos dietéticos están ocupando posiciones de centralidad en el entramado biomédico de intervención sobre el problema. 
Lo mismo sucede con las posibles soluciones para el sobrepeso, donde las opciones doméstico-familiares de corte nutricional: "seguir una dieta equilibrada", "hacer cinco comidas al día", "consumir frutas y verduras", predominan sobre otros ámbitos de actuación colectivos. Así, pocos padres hicieron mención a medidas relacionadas con la intervención de la administración, como el trabajo desde ayuntamientos, centros de salud o colegios.

Puede entenderse que la repetición de posibles soluciones como: "conocer la pirámide nutricional", "seguir la dieta mediterránea" o "enseñar a comer a nuestros hijos" viene a mostrar la influencia acrítica de los discursos expertos, de origen biomédico y nutricional, en unos padres sometidos a un bombardeo de información que termina por responsabilizarlos del problema desde concepciones que no ofrecen soluciones más allá de lo individual.

Hay que señalar que, cuando el manejo de la conversación grupal se dirigió a la importancia de la participación de actores colectivos que van más allá del ámbito sanitario, comentarios como: "la necesidad de una mayor formación e información", "el cuidado de la calidad alimentaria", "un mejor etiquetado alimentario" o la necesidad de "una mayor conciencia social" eran referencias pasivas que parecían demandar acciones y responsabilidades sin saber muy bien en qué consistirían, probablemente debido a la escasa tradición participativa de los sistemas públicos en España en general, y específicamente del sistema sanitario y asistencial $^{25}$.

\section{CONCLUSIÓN}

Las percepciones que presentan los niños/as sobre la obesidad y el sobrepeso son predominantemente estéticas y relacionadas con aspectos de su sociabilidad. Por su parte, los padres presentan una fuerte preocupación por los costes sociales de integración y las consecuencias psicológicas para sus hijos, percibiendo la obesidad como un problema de salud más grave y lejano. En cuanto al género, se cuestionan ciertos matices relacionados a los efectos psicosociales del sobrepeso en niños y niñas, a la par que se intuye una tendencia homogeneizadora incipiente, también percibida por los padres.

Respecto del cuestionamiento por el tipo de soluciones que se están proponiendo actualmente, entre los padres predomina una concepción nutricional del sobrepeso y la obesidad junto al desconocimiento del papel que deberían jugar instituciones públicas no necesariamente sanitarias. El principal límite que las formas de prevención deben abordar, además de diversificar sus enfoques hacia segmentos poblacionales más $\operatorname{concretos}^{26}$ - por ejemplo introduciendo la diversidad de sexo -, es precisamente la individuación del sobrepeso y sus causas, que hace que no sea visto como un problema de todos sino de quienes lo padecen, dificultando hacerlos partícipes de las posibles soluciones.

\section{COLABORADORES}

J NAVAS LÓPEZ y J PALACIOS RAMÍREZ participaron de concepción y diseño de la investigación, análisis e interpretación de los datos y redacción del artículo. A ROCA GEA participado de recogida y análisis de los datos.

\section{REFERENCIAS}

1. Organización Mundial de la Salud. Estrategia sobre régimen alimentario, actividad física y salud. Géneva: OMS; 2004.

2. Padilla I. Prevalencia de sobrepeso-obesidad y factores asociados con valor predictivo-preventivo en escolares de 6 a 11 años de Río Gallegos, Santa Cruz, Argentina. Salud Colect. 2011; 7(3):377-88.

3. Burrows A, Vivien Z, Leiba B, Barrera A, Burgueño A. Características biológicas, familiares y metabólicas de la obesidad infantil y juvenil. Rev Méd Chile. 2001; 129(10):1155-62. http:// dx.doi.org/10.3305/nh.2013.28.2.6280

4. Aranceta J, Pérez C, Ribas L, Serra L. Epidemiología y factores determinantes de la obesidad infantil y 
juvenil en España. Rev Pediatr Aten Primaria. 2005; 7(1):13-20.

5. Zonana-Nacah A, Conde Gaxiola ME. Mother's perception of their children's obesity. Gac Med Mex. 2010; 146(3):165-68.

6. Evans WD, Finkelstein EA, Kamerow DB, Renaud JM. Public perceptions of childhood obesity. Am J Prev Med. 2005; 28(1):26-32.

7. Wilson LF. Adolescents'attitudes about obesity and what they want in obesity prevention programs. J Sch Nurs. 2007; 23(4):229-38.

8. Fonseca H, Gaspar de Matos M. Perception of overweight and obesity among Portuguese adolescents: An overview of associated factors. Eur J Public Health. 2005; 15(3):323-8.

9. Carole RC, Cynthia J, Cyntia LF, Gregory JJ, Cid CS. The perception of obesity by normal-weight versus obese school-age children. Child Psychiatry Hum Dev. 1986; 17(2):113-20.

10. Hammersley M, Atkinson P. Etnografía: métodos de investigación. Barcelona: Paidós; 1994.

11. Werner O, Schoepfle M. Systematic fieldwork. London: Sage; 1985. Vol. 2.

12. Osuna-Ramirez I, Hernández-Prado B, Campuzano J, Salmerón J. Indice de masa corporal y percepción de la imagen corporal en una población adulta mexicana: la precisión del autorreporte. Salud Pública Méx. 2006; 48(2):94-103 [citado 2013 jun 1]. Disponible en: http://www.scielo.org.mx/ scielo.php?script=sci_arttext\&

13. Núñez HP. Las creencias sobre obesidad de estudiantes de la Educación General Básica. Rev Educ. 2007; 31(1):145-64.

14. Montero JC. Obesidad: una visión antropológica [maestría]. Buenos Aires: Universidad de Buenos Aires; 2001.

15. Vázquez R, López X, Álvarez G, Mancilla JM, Oliva A. Insatisfacción corporal e influencia de los modelos estéticos en niños y jóvenes varones mexicanos. Enseñ Invest Psicol. 2006; 11(1):185-97.

16. Gandarillas A, Bru S, Sepúlveda AR, Galán I, Díez L, Zorrilla B. Autopercepción de peso, índice de masa corporal y conductas relacionadas en adolescentes. Gac Sanit. 2005; 19(1):13.

17. Fairburn CG, Doll HA, Welch SL, Hay PJ, Davies BA, O'Connor ME. Risk factors for binge eating disorder. A community based case-control study. Arch Gen Psychiatry. 1998; 55(5):425-32.

18. Triches RM, Giugliani ERJ. Insatisfação corporal em escolares de dois municípios da região Sul do Brasil. Rev Nutr. 2007; 20(2):119-28. http://dx.doi.org/ 10.1590/S1415-52732007000200001

19. Alves D, Pinto M, Alves S, Mota A, Leirós V. Cultura e imagem corporal. Motricidade. 2009; 5(1):1-20.

20. Carvalho MC, Martins A. A obesidade como objeto complexo: uma abordagem filosófico-conceitual. Ciênc Saúde Colet. 2004; 9(4):1003-12.

21. Donzelot J. La policía de las familias. Barcelona: Pre-Textos; 1990.

22. Salas-Salvadó J, Rubio MA, Barbany M, Moreno B, Grupo Colaborativo de la SEEDO. Consenso SEEDO 2007 para la evaluación del sobrepeso y la obesidad y el establecimiento de criterios de intervención terapéutica. Med Clin. 2007; 128(5):184-96.

23. Rose $N$. The politics of life itself: Biomedicine, power, and subjectivity in the twenty-first century. Princeton: Princeton University Press; 2006. http:// dx.doi.org/10.1002/jhbs.20402

24. Rose N, Miller P. Political power beyond the state: problematics of government. Br J Sociol. 1992; 43(2):173-205.

25. Navarro V. El subdesarrollo social de España: causas y consecuencias. Barcelona: Anagrama; 2009.

26. Campos LA, Leite AJM, Almeida PC. Nível socioeconômico e sua influência sobre a prevalência de sobrepeso e obesidade em escolares adolescentes do município de Fortaleza. Rev Nutr. 2006; 19(5):531-8. http://dx.doi.org/10.1590/S1415-52 732006000500001

Recibido: Diciembre 12, 2013 Versión final: Noviembre 5, 2014 Aprobado: Noviembre 17, 2014 
